\title{
The Cruise Missile Guidance and Control System Design and Simulation Based on the Integral Sliding Mode Control
}

\author{
Liu Xipeng, a \\ ${ }^{1}$ Shanghai University of Finance and Economics, China \\ aaxi853@163.com
}

Keywords: Integral Sliding Mode Control, Cruise Missile, Guidance and Control System, MATLAB Abstract. As a long-range precision guided high-tech weaponry, the cruise missiles have the advantages of strong penetration prevention capability, diversification of tactical use, and economical efficiency etc. After the Gulf War, many countries competed for research and develop the cruise missile. In this paper, the cruise missile's guidance system and control system are designed separately. The proportional guidance method is used as the guidance system guidance method. The PID control and integral sliding mode control are used to design the controller. The simulation results show that the integral sliding mode control can effectively improve the guidance accuracy and rapid strike ability of cruise missiles, and provide a new method for missile control.

\section{Introduction}

The cruise missile is a missile that relies on the thrust of jet engine and the aerodynamic lift of a wing to fly in a dense atmosphere mainly in a cruising state [1]. At present, various countries have developed missiles in the direction of stealth, increased range and cruising speed based on the shortcomings and problems exposed by cruise missiles in actual combat, and they have the ability to intelligently analyze, evaluate and coordinate their offensive capabilities. Therefore, by studying the guidance and control system of cruise missiles to improve the penetration ability and combat capability, it has the great significance both in theory and application.

Missile guidance control technology is the key technology to promote the development of missiles. It involves the entire life cycle of missile design, guidance and application, and in a large extent determines the tactical and technical performance of missiles [2]. The basic function of the guidance system is during missile strikes, measure the relative motion of the missile and target, and obtain guidance instructions required by the guidance law in order to automatically control the missile correction deviation which is the "guide" function of missile. The basic function of the control system is to form control commands according to a certain control law based on the generated guidance instructions and the missile's own movement state, controlling the missile's actuator to generate corresponding control forces and moments to change the missile's flight status and ensure the missile's stable flight and accurately hit the target, this is the "control" function of missile, which composition is shown in Figure 1.

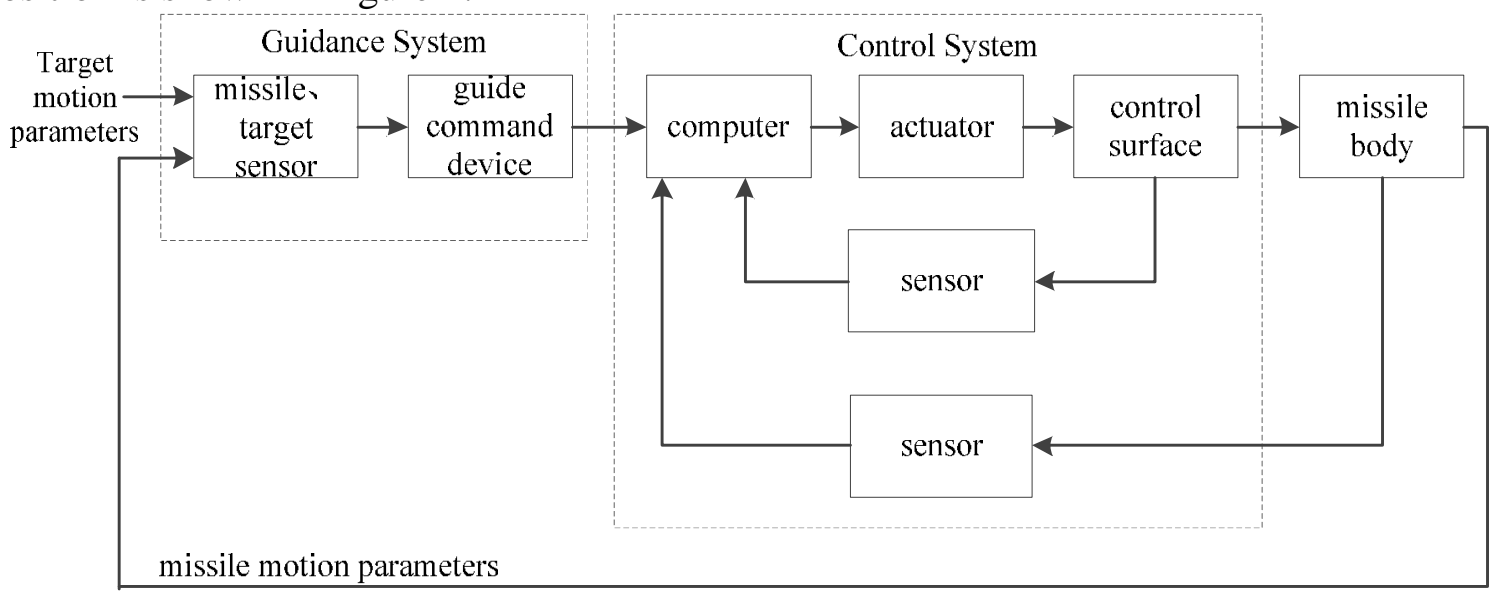

Fig.1 Block diagram of missile guidance control system 
The requirements of the guidance control system are that the highly guidance accuracy, the resolution of the target is strong, the reaction time is as short as possible, the largely control capacity, the stronger anti-jamming capability and the highly reliability and maintainability [3]. This paper designed a cruise missile guidance control system to compare missile performance under different controllers.

\section{Designing missile guidance control system}

The mathematical model of the cruise missile is composed of the equations of motion, including the dynamic equations of the mass center's movement and the attitude transformation of the missile, the kinematics equation, the mass change equation, the angle geometric relation equation and the control relation equation, etc., the specific equations are detailed in [4]. In this paper, the guided missile control system is designed for the submarine section of the cruise missile. Firstly, the guidance system is designed, including the guidance law and guidance strategy. Then the control system is designed and the overload control is used as the missile control method. The controller design uses an integral sliding mode control (SMC) algorithm, and finally compares the missile guidance control effect of the PID controller and the integral SMC controller.

\section{Designing guidance system}

In this paper used the gravity-compensated proportion method to guidance the terminal missile, used bank-to-turn approach to control missile, which achieved the fastest maneuvering of missile requires a roll-pitch hybrid maneuver to achieve. Therefore, during the flight of the missile, the rolling channel rapidly turns the maximum lift surface of the missile to the ideal maneuvering direction; the pitch channel acquires missiles at lift surface and receiving need-overload. Suppose the components of the target line-of-sight rotation angular velocity on the $\mathrm{y}$ and $\mathrm{z}$ axes of the missile's non-scrolling coordinate system are $\delta_{y}^{\mathcal{\alpha}}$ and $\delta_{z}^{\alpha}, n_{y h c}$ and $n_{z h c}$ are the overload instructions of the missile on the $\mathrm{y}$ and $\mathrm{z}$ axes of the missile's non-scrolling coordinate system, hence, the guidance is obtained. The model is:

$$
\left\{\begin{array}{l}
n_{y h c}=\frac{K \mid \mathcal{R}^{\mathcal{E}}}{g} \delta_{z}^{\alpha}+\cos \theta \\
n_{z h c}=-\frac{K \mid \mathcal{R}^{\mathcal{\alpha}}}{g} \delta_{y}^{\alpha}
\end{array} .\right.
$$

Among them $K$ is the guidance ratio coefficient, $\theta$ is the ballistic inclination of the missile, $n_{y h c}$ and $n_{z h c}$ are the expected instruction of the missile guidance controller.

This paper used overload control to design guidance control system, missile control instructions are divided into roll command $\phi_{c}$, pitch channel overload command $n_{y c}$ and yaw channel overload command $n_{z c}$, using the gravity compensation proportional guidance law to guide, using the following guidance strategy to form the missile maneuver instruction $n_{y c}, n_{z c}$ and roll instruction $\phi_{c}$ :

$$
\left\{\begin{array}{l}
\phi_{c}^{\prime}=\arctan \left(\frac{n_{z h c}}{n_{y h c}}\right) \times 57.3 \\
\boldsymbol{V}_{c}^{\prime}=\phi_{c}^{\prime}-\phi \\
\phi_{c}=\arctan \left(\frac{n_{z h c}}{n_{y h c}}\right) \times 57.3+K_{1} \times 180^{\circ} . \\
n_{y c}=\left(n_{y h c}^{2}+n_{z h c}^{2}\right)^{1 / 2} \cdot M R E \\
n_{z c}=0
\end{array}\right.
$$


Where $\phi$ is the actual rolling angle of the missile; $\phi_{c}^{\prime}$ is the given roll angle of the guiding law; $V \phi_{c}^{\prime}$ is the roll angle error; $K_{1}$ is the minimum roll angle adjustment coefficient, the value is $K_{1}=0, \pm 1, \pm 2$; $M R E$ is the sign variable, and the value is $M R E= \pm 1[5]$.

\section{Designing control system}

Using overload control as the cruise missile control system, the overload control scheme adopted is to use the deviation of the missile overload to design the control law of the rudder angle, just as:

$$
\delta_{r}(t)=k_{n_{z}}\left(n_{z c}-n_{z}\right)=k_{n_{z}} \Delta n_{z} .
$$

Where $\delta_{r}$ is the rudder deflection angle command, $n_{z c}$ is the desired overload of the yaw channel, and $n_{z}$ is the actual overload.

For instance, an overload-controlled missile longitudinal channel stabilization loop is shown in Figure 2.

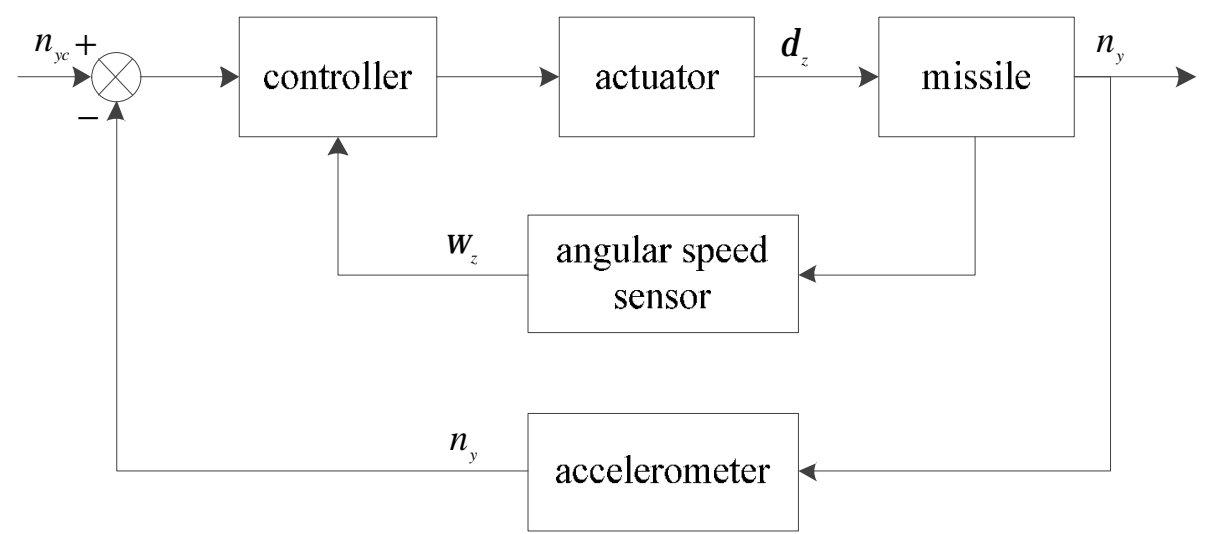

Fig.2 Block diagram of longitudinal channel stabilization loop

From Figure 2 we can see that when the missile's overload control directly controls the longitudinal channel overload and the control of the missile's angular velocity, the longitudinal overload directly reflects the maneuvering capability of the missile, and this solution has a better control effect to achieve the end maneuver of the missile [6].

The controllers in Fig.2 are designed using PID control and integral SMC control, in this paper we focus on the designing of the integral SMC controller. Since overload control is required to measure the missile's overload and angular velocity, but the derivative of the overload has no physical meaning, thus the integral type sliding mode surface is used to design the sliding mode controller, using integration which has a strong self-adaptive ability and can eliminate many uncertain errors while modeled system.

Because the missile flight in the vertical plane, we only designed the integral SMC controller in the missile's longitudinal channel. The overload control needs angular velocity feedback compensation, therefore, the pitch angle velocity $\omega_{z}$ is introduced, so the following hyper plane is selected as the sliding mode surface:

$$
s=\mathrm{c}_{1}\left(n_{y c}-n_{y}\right)+\mathrm{c}_{2} \int\left(n_{y c}-n_{y}\right) d t+\mathrm{c}_{3}\left(\omega_{z c}-\omega_{z}\right)+\mathrm{c}_{4} \int\left(\omega_{z c}-\omega_{z}\right) d t
$$

Where $c_{1}, c_{2}, c_{3}, c_{4}$ are greater than zero, $n_{v c}, \omega_{z c}$ are the desired overload and pitch rate, and approximate as $\omega_{z c}=\omega_{z c}=0$.

The final pitch channel overload control law obtained by derivation is as follows, which parameter values can refer to literature [7].

$$
u=\left[-\varepsilon \operatorname{sgn}(s)-k s-\left(\mathrm{I}_{1} \omega_{z}+\mathrm{I}_{2}+\mathrm{I}_{3} \delta_{z}+\mathrm{I}_{4} n_{y}\right)\right] / \mathrm{I}_{5} .
$$




\section{Simulation and analysis}

\section{System simulation model}

In this paper the designing of simulation platform is based on MATLAB simulation, using a modular design method to establish a system simulation model, as shown below:

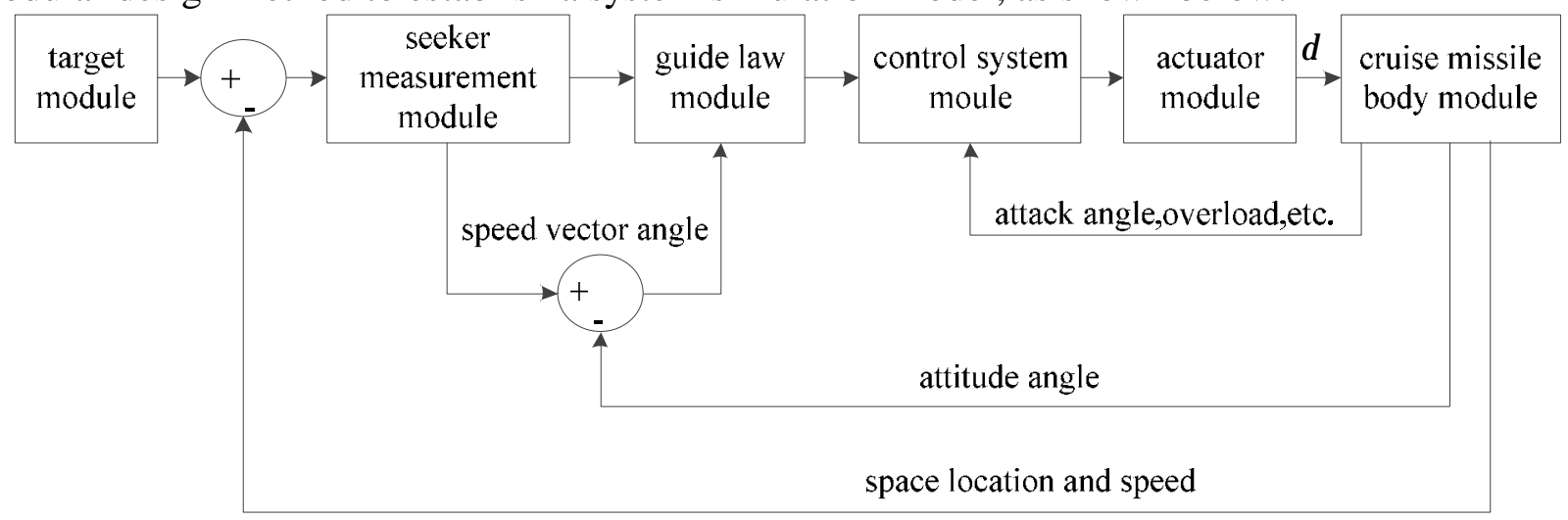

Fig.3 System simulation module diagram

After build all the modules, we simulate the cruise missile guidance and control system.

\section{Simulation}

The initial conditions of the simulation: the strike target is a static target, its position coordinate is $(7000,0,0)$ and the initial position of the cruise missile is $(0,1000,0)$ which means the height of missile is 1000 meter, the initial speed of the missile of $400 \mathrm{~m} / \mathrm{s}$. The initial attack angle equation is $\alpha_{0}=0^{\circ}$, the initial pitch angle $\theta_{0}=0^{\circ}$, the initial yaw angle $\psi_{0}=0^{\circ}$, the initial roll angle $\phi_{0}=0^{\circ}$, the initial side slip angle $\beta_{0}=0^{\circ}$, the initial ballistic deflection angle $\psi_{\mathrm{a} 0}=0^{\circ}$ and the ballistic inclination angle $\theta_{\mathrm{a} 0}=0^{\circ}$.

Firstly, we simulated the missile system under the PID controller, the results shown as Figure 4:
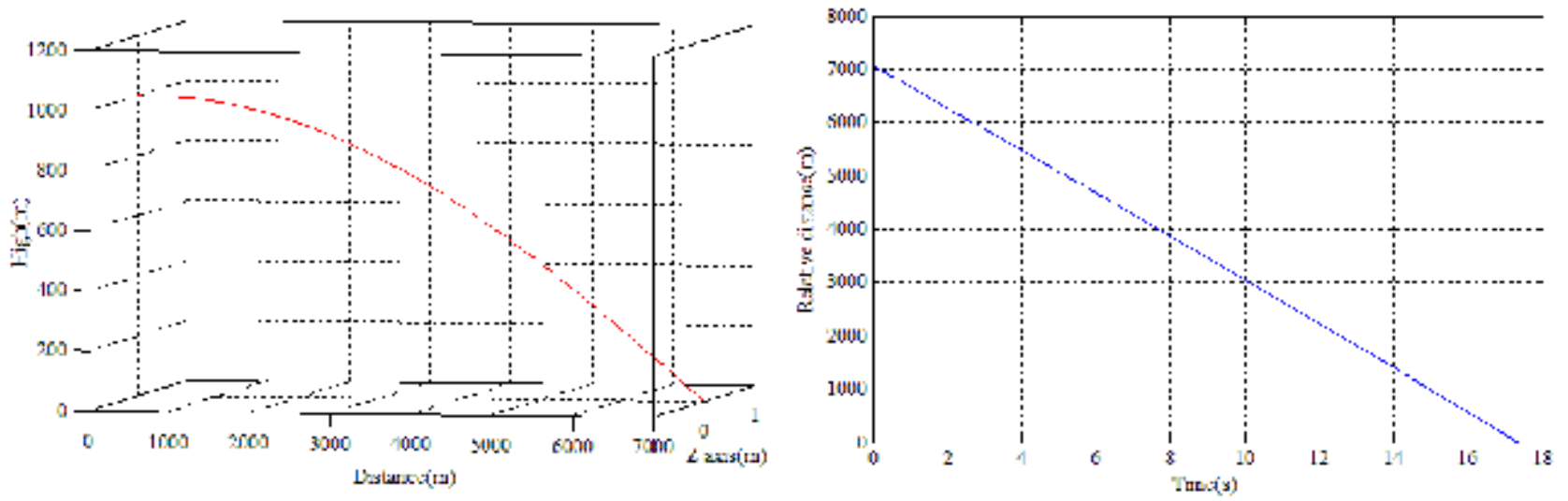

Fig. 4 the PID controller controlled missile motion track

The left graph of Figure 4 describes the ballistic trajectory of the cruise missile using the PID controller to strike a static target, which shown it can be used to achieve missile guidance and control, and ultimately strike target accurately. The right graph of Figure 4 shows the missile's relative distance curve with the target, from the result we can obtain the miss distance is 0.88 meters and the flight time is 17.35 seconds.

Secondly, we simulated the system under the integral SMC controller, the results shown as Figure 5 , the left graph shows the ballistic trajectory of the cruise missile using the integral SMC controller, the right graph shows the relative distance of missile and target, the result of the miss distance is 0.001 meters and the flight time is 17.34 seconds. 

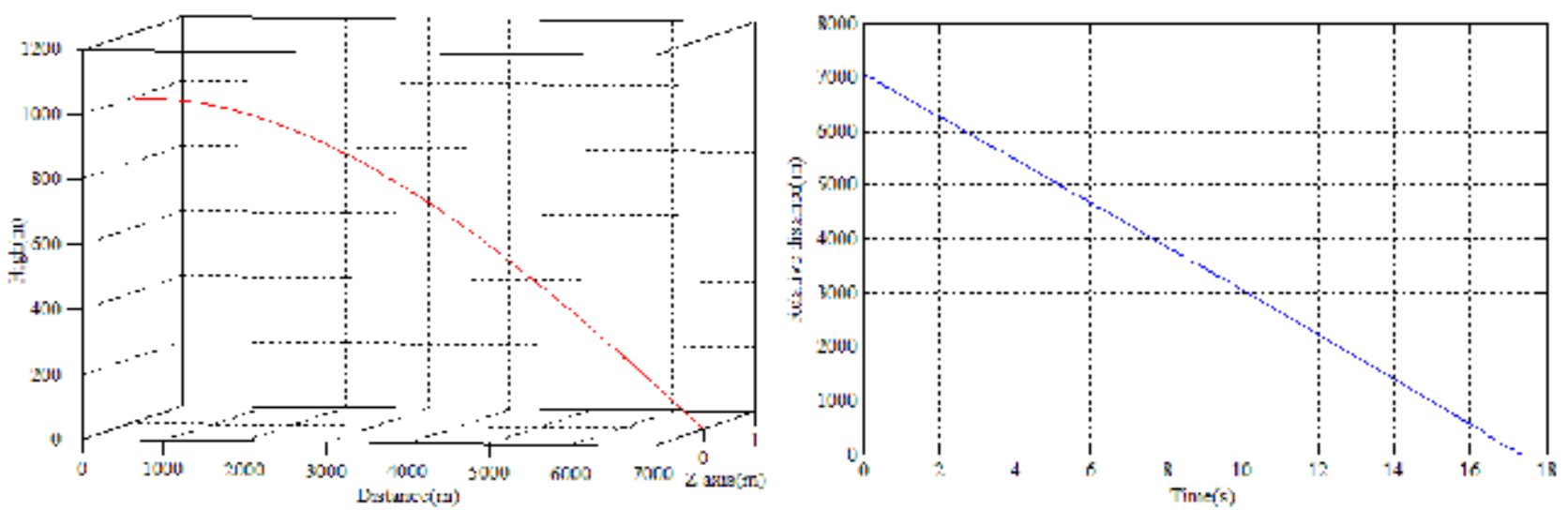

Fig.5 the integral SMC controlled missile motion track

\section{Simulation analysis}

The accuracy of missile guidance is the most significant indicator of the guidance system, it is usually expressed as the miss distance. In this paper, the miss distance is expressed as the absolute distance between the missile's center and the target's center while the missile hits the ground moment. The rapid strike capability is the flight time required to guide the guidance of missile, the shorter flight time, the faster attacking speed, which means the smaller rate of being intercepted by the enemy.

Therefore, throught the above-mentioned simulation, it can be seen that the missile designed using the integral SMC controller has better missile guidance accuracy and rapid strike capability.

\section{Conclusions}

This paper used the guidance control system of the cruise missile as a model, designing the guidance system and the control system. The PID controller and the integral SMC controller are mainly introduced to control the cruise missile. From the results, it can be seen that the integral SMC controller has better missile guidance accuracy and rapid attack capability. Therefore, it contributes to improving the performance of controller designing of the cruise missile.

\section{Acknowledgements}

This work was financially supported by the National Natural Science Foundation (71472113) and the Fundamental Research Funds for the Central Universities(2017110757).

\section{References}

[1] Qi ZK, Xia QL. Guided weapon control systems. Beijing Institute of Technology (2004).

[2] Fleeman L Eugene. Tactical Missile Design (Second Edition). Reston: American Institute of Aeronautics and Astronautics (2006).

[3] Yang Jun. Missile Control System Design Principle. Northwestern Polytechnic University Publishers (1997).

[4] Qian XF, Lin RX, Zhao YN. Missile Flight Mechanics. Beijing Institute of Technology Publisher (2011).

[5] Tang YH. BTT Missile Robust $H_{\infty}$ Autopilot Design. Northwestern Polytechnic University (2004).

[6] Chen XJ. Research on the realization of terminal maneuvering trajectory for anti-ship missile. Harbin Engineering University (2007).

[7] Liu XP. Multi-dimensional Taylor network optimal control of the plane symmetrical cruise missile flight for attacking static target. Southeast University (2017). 